\title{
Reductive iron uptake by Candida albicans: role of copper, iron and the TUP1 regulator
}

\author{
Simon A. B. Knight, ${ }^{1}$ Emmanuel Lesuisse, ${ }^{2}$ Robert Stearman, ${ }^{3}$ \\ Richard D. Klausner ${ }^{3}$ and Andrew Dancis ${ }^{1}$
}

Author for correspondence: Andrew Dancis. Tel: +1215 573 6275. Fax: +1215 5737049.

e-mail: adancis@mail.med.upenn.edu

\footnotetext{
1 Department of Medicine, Division of

Hematology-Oncology, University of Pennsylvania, Philadelphia, PA 19104, USA

2 Laboratoire d'Ingénierie des Protéines et Contrôle Métabolique, Institut Jacques Monod, Tour 43, Université Paris 7/Paris 6, 2 Place Jussieu, 75251 Paris Cedex 05, France

3 Office of the Director, $\mathrm{NCl}$, National Institutes of Health, Bethesda, MD 20892, USA
}

\begin{abstract}
High-affinity iron uptake by a ferrous permease in the opportunistic pathogen Candida albicans is required for virulence. Here this iron uptake system has been characterized by investigating three distinct activities: an externally directed surface ferric reductase, a membrane-associated PPD ( $p$ phenylenediamine) oxidase and a cellular ferrous iron transport activity. Copper was required for the PPD oxidase and ferrous transport activities. In contrast, copper was not required for iron uptake from siderophores. Addition of iron to the growth medium repressed ferric reductase and ferrous transport, indicating homeostatic regulation. To identify the genes involved, orthologous mutants of Saccharomyces cerevisiae were transformed with a genomic library of C. albicans. CFL95, a gene with sequence similarity to ferric reductases, restored reductase activity to the orthologous S. cerevisiae mutant. CaFTR2 and CaFTR1, genes with homology to ferrous permeases, conferred ferrous transport activity to the orthologous S. cerevisiae mutant. However, neither a genomic library nor CaFET99, a multicopper oxidase homologue and candidate gene for the PPD oxidase, complemented the S. cerevisiae mutant, possibly because of problems with targeting or assembly. Transcripts for CFL95, CaFTR1 and CaFET99 were strongly repressed by iron, whereas the CaFTR2 transcript was induced by iron. Deletion of the TUP1 regulator perturbed the homeostatic control of reductive iron uptake. Incidentally, iron starvation was noted to induce flavin production and this was misregulated in the absence of TUP1 control. The opposite regulation of two iron permease genes and the role of TUP1 indicate that the process of iron acquisition by C. albicans may be more complex and potentially more adaptable than by $S$. cerevisiae.
\end{abstract}

Keywords: iron regulation, ferric reductase, multicopper oxidase, ferrous permease, siderophores

\section{INTRODUCTION}

Candida albicans is a fungal commensal that inhabits mucosal surfaces, such as the mucosa of the gastrointestinal tract. In certain clinical situations, C. albicans may become virulent, invading locally and disseminating via the bloodstream (Fridkin \& Jarvis, 1996). When granulocyte counts are low, such as following bone marrow transplantation, chemotherapy or in patients with AIDS, the risk of infection is extremely high and the infections are difficult to eradicate (Warnock, 1998).

\footnotetext{
Abbreviations: BCS, bathocuproinedisulfonate; BPS, bathophenanthrolinedisulfonate; CSM, complete synthetic medium; PGK, phosphoglyerol kinase; PPD, $p$-phenylenediamine.
}

In infected tissue, C. albicans proliferates in both yeast and filamentous forms. The filamentous morphology is repressed by the TUP1 regulator (Braun \& Johnson, 1997) and may play a role in invasiveness.

Virulence of many pathogens requires expression of specific iron uptake systems, perhaps to counter ironwithholding host defences that operate in different environmental niches (Ratledge \& Dover, 2000). A recent report implicates a plasma membrane component of a high-affinity iron uptake system in virulence of $C$. albicans (Ramanan \& Wang, 2000). Here we characterize this iron acquisition system using the related organism, Saccharomyces cerevisiae. In this related yeast, the initial step in iron uptake is mediated by an externally directed reductase. (Dancis et al., 1990). The 
genes responsible for this activity, FRE1 and FRE2, encode membrane-associated b-type haem proteins (Shatwell et al., 1996). The ferrous iron produced by the reductase in turn is captured and transported across the plasma membrane by a protein complex. The components of this complex are a multicopper oxidase homologous to ceruloplasmin (encoded by FET3) (Askwith et al., 1994) and a polytopic membrane permease (encoded by FTR1) (Stearman et al., 1996). In $S$. cerevisiae, interference with copper delivery to the oxidase abrogates high-affinity iron uptake from ferric chelates (Dancis et al., 1994). Each of these components, the reductase, the oxidase and the permease are individually required for iron acquisition from ferric chelates and the entire system is homeostatically regulated in response to available iron with induction occurring in response to iron starvation (Askwith et al., 1996).

Here we show that in C. albicans similar iron acquisition activities are present - ferric reductase, PPD ( $p$ phenylenediamine) oxidase and ferrous transport. We show that, as in S. cerevisiae, copper is needed for the oxidase and iron transport activities. Complementation of mutants of S. cerevisiae was used to identify orthologous genes from C. albicans, and regulation of reductive iron uptake was examined by biochemical assays and Northern blotting with candidate genes for ferric reductase, oxidase and ferrous permease. Iron exposure during growth has major effects, and TUP1, a global regulator of morphology and metabolism, also plays a major role in iron-dependent gene regulation. A separate copper-independent system for efficient iron uptake from siderophores exists in C. albicans (Lesuisse et al., 2002). Overall our results suggest that the complexity of the C. albicans reductive iron uptake system is greater than in baker's yeast.

\section{METHODS}

Strains. S. cerevisiae strain 499 $\Delta 1 \Delta 2$ (MATa ura3-52 lys2-801 ade2-101 trp1- $\Delta 63 \Delta$ fre 1::LEU2 $\Delta$ fre2::HIS3) lacking ORFs for FRE1 and FRE2 (Finegold et al., 1996) was used for complementation cloning of CFL95. To clone CaFTR2, S. cerevisiae strain 42-3C1 (MATa ura3-52 lys2-801 ade2-101 his3- 200 leu2- $\Delta 1$ ftr1 $1:: T R P 1)$, in which the entire FTR1 ORF was replaced with TRP1, was used (Stearman et al., 1996). C. albicans strains used were SC5314 (wildtype), CBS5737 (wild-type, ATCC 32032) and CAI4 (ura3s::imm434/ura3s::imm434) (Fonzi \& Irwin, 1993). BCa02-10 (tup1s:: hisG/tup1s:: hisG::p405-URA3, ura3/ ura3) was derived from CAI4 and was a gift from B. Braun and A. Johnson (Braun \& Johnson, 1997).

Media and growth conditions. S. cerevisiae and C. albicans were grown in YPD supplemented with $20 \mathrm{mg}$ adenine $\mathrm{l}^{-1}$ (YPAD) at $30^{\circ} \mathrm{C}$. Defined growth medium for growth of $\mathrm{C}$. albicans consisted of complete synthetic medium (CSM)uracil (Bio101) supplemented with $100 \mathrm{mg}$ uridine $\mathrm{l}^{-1}, 6.7 \mathrm{~g}$ yeast nitrogen base without amino acids $1^{-1}$ (Difco) and $2 \%$ D-glucose. Amino acids and uridine were omitted from the defined medium as required for selecting plasmids. The ironlimited defined medium contained yeast nitrogen base lacking iron and copper (Bio101). Copper sulfate and ferric ammo- nium sulfate were added as required. The iron chelator bathophenanthrolinedisulfonate (BPS) or the copper chelator bathocuproinedisulfonate (BCS) were added as noted to limit availability of iron or copper, respectively.

PCR and plasmid construction. The ORFs of CFL95, CaFTR1, CaFTR2 and CaFET99 were amplified by PCR with the following oligonucleotide pairs. Genomic sequence is shown in upper case letters and flanking linkers are shown in lower case letters.

CFL95: 5'-tagagctcATGGTAGCTATCAATTCATTATTATTTGCTGCC-3' and 5'-taggatccCTACCAGCTTTGCATTTGTTCAAACAATTCAACTCTG-3'

CaFTR $1: 5^{\prime}$-tagagctcATGGTTGACGTATTTAACGTTCAAATTTTC-3' and 5' ${ }^{\prime}$-taggatccTTATTTGTTTTCTTTGGATTCGATCAATTTG-3'

CaFTR2: 5'-tagagctcATGGTTGATGTTTTCAATGTTCAAGTC-3' and '5'-taggatccTTATTTATTTTCTTGAGTTTCGACTAATTTG-3'

CaFET99: 5'-tagagctcATGCGGTTTATTGTATCATCATTTATATTTTTTATCTC-3' and $5^{\prime}$ taggatccCTAATGTTGTTTAGAGTTACTTCCAGAAGAAGAGG-3'

Genomic DNA isolated from C. albicans SC5314 was used as the template and a combination of polymerases, Pfu-Turbo (Stratagene) and Taq (Promega) in a 4:1 ratio was added. The PCR products were cloned into pCRII-TOPO. The plasmids generated were termed pTOPO-CFL95, pTOPO-CaFTR1, pTOPO-CaFTR2 and pTOPO-CaFET99. The DNA sequences were compared to sequences of clones isolated from the genomic library, p1A2 (CFL95) and pBS1996 (CaFTR2), and to Contig 6 of the C. albicans genome from the Stanford DNA Sequencing and Technology Center (http://wwwsequence.stanford.edu/group/candida). The ORFs isolated from the pCRII-TOPO vectors by digestion with SstI and BamHI were ligated into the corresponding sites of the $S$. cerevisiae expression vector YIpDCE1 (Stearman et al., 1998), yielding plasmids pDC-CFL95, pDC-CaFTR1, pDC-CaFTR2 and pDC-CaFET99. Two CUG codons in the coding region of CaFET99 in pDC-CaFET99 were converted by site-directed mutagenesis (Quick Change; Stratagene) to AGC and TCT, respectively, creating pDC-CaFET99-Ser. For co-expression of CaFET99 with CaFTR1, the ORF for CaFTR1 was subcloned from pTOPO-CaFTR1 into the second multiple cloning linker of pDC-CaFET99-Ser between AvaI and AvrII. For co-expression of CaFTR2 with CaFET99, the ORF for CaFTR 2 was inserted into the second multiple cloning site of pDC-CaFET99-Ser between AvrII and XhoI.

Transformation of $S$. cerevisiae with a $C$. albicans genomic DNA library. The C. albicans genomic library for complementation of $S$. cerevisiae was a gift from Gerald Fink, Whitehead Institute (Liu et al., 1994). S. cerevisiae was transformed with the library using electroporation (Becker \& Guarente, 1991). For transformation of individual plasmids, the lithium acetate method was used (Agatep et al., 1998). p1A2 containing CFL95 was cloned by selecting $S$. cerevisiae $499 \Delta 1 \Delta 2$ transformants on specially designed medium. The medium included $\left(1^{-1}\right): 6.7$ g yeast nitrogen base without iron or copper (Bio101), 0.8 g CSM-ura (Bio101), $20 \mathrm{~g}$ D-glucose, $25 \mathrm{mM}$ MES, pH 6.1 (Sigma), $15 \mathrm{~g}$ agarose (Fluka) and $1 \mathrm{mM}$ ferrozine (Fluka). Strain $499 \Delta 1 \Delta 2$ does not grow on this medium. Selected transformants were assayed for ferric reductase activity (see below). pBS1996 containing CaFTR2 was cloned by selecting strain 42-3C1 ( $\Delta f t r 1)$ transformants on low-iron medium (Stearman et al., 1996). The $\Delta f t r 1 S$. cerevisiae strain grows slowly on this medium. Plasmids were 
rescued from more rapidly growing transformants and retransformed into E. coli strain $\mathrm{DH} 5 \alpha$ for large-scale plasmid preparations.

Ferric reductase, high-affinity ferrous uptake, ferrichrome uptake and PPD oxidase assays. The assays for ferric reductase have been described previously (Dancis et al., 1990) and rely on the formation of a coloured BPS-Fe(II) complex. High-affinity ferrous iron uptake was measured as described previously (Dancis et al., 1994). Equal volumes of ferrichrome and ferric chloride $(260 \mu \mathrm{M}$ each in water) were incubated for $5 \mathrm{~min}$ at room temperature after which $1 \mathrm{M}$ Tris/ $\mathrm{HCl}$ buffer, $\mathrm{pH} 7 \cdot 4$, was added to give a final iron-siderophore concentration of $100 \mu \mathrm{M}$. The final concentration of ${ }^{55} \mathrm{Fe}$-labelled siderophore used for iron uptake assays was $1 \mu \mathrm{M}$. Ferric reductase and ferrous uptake activities were expressed per mg total cellular protein in reporting experiments involving filamentous cells. Protein levels in yeast or filamentous forms of $C$. albicans were determined by solubilizing in Y-PER reagent (Pierce) and quantifying the soluble protein (BCA; Pierce).

To assay multicopper oxidase activity, a cell membrane fraction was prepared from exponentially grown cells as described by Yuan et al. (1995). Oxidase activity was detected using a gel-based assay (Yuan et al., 1995) or liquid assay (Spizzo et al., 1997).

RNA analysis. Cells or hyphae from $100 \mathrm{ml}$ cultures were washed in buffer (50 mM citrate, $\mathrm{pH} 6 \cdot 6,5 \%$ dextrose) and total RNA was isolated by extraction with hot acidic phenol. After separation of $30 \mu \mathrm{g}$ aliquots on formaldehyde gels, RNAs were transferred to nitrocellulose membranes (Schleichler \& Schuell) by capillary blotting. Probes for CaFTR1, CaFTR2, CFL1, CaFET3, CaFET99 were generated using primer pairs to amplify approximately $100 \mathrm{bp}$ of region immediately $3^{\prime}$ of the stop codon for each gene. We selected the non-coding regions because these provided unique probes for each of these genes. Genomic DNA from SC5314 was used as template for the PCR reactions and the products were isolated from polyacrylamide gels. The CFL95 probe was a $1 \cdot 1 \mathrm{~kb}$ HindIII-BglII fragment isolated from plasmid p1A2. The ACT1 probe was a $1 \cdot 1 \mathrm{~kb}$ fragment containing the ACT1 ORF of $S$. cerevisiae. All probes were labelled with ${ }^{32} \mathrm{P}$ radionuclide by random priming (Gibco-BRL). Prehybridization and hybridization were carried out in $50 \%$ formamide at $42{ }^{\circ} \mathrm{C}$. Blots were washed with $0 \cdot 2 \times \mathrm{SSC} / 0.1 \%$ SDS at room temperature followed by washes at $42{ }^{\circ} \mathrm{C}$. PhosphorImage screens were exposed for $72 \mathrm{~h}$ with the exception of the CaFET99 blot which was exposed for $6 \mathrm{~d}$. Images obtained from the PhosphorImager (Storm 860; Molecular Dynamics) were adjusted for contrast using the levels tool in Adobe Photoshop.

Flavin analysis. Supernatants from stationary-phase C. albicans cultures were obtained by centrifugation. Spectra for each supernatant were obtained between 550 and $300 \mathrm{~nm}$ using an Olis modified Cary 14 spectrophotometer. Flavins were quantified at the absorption maximum of $446 \mathrm{~nm}$ against a calibration curve constructed from a riboflavin standard.

\section{RESULTS}

\section{Cell-surface ferric reductase activity}

Cell-surface ferric reductase activities of two reference strains of C. albicans, SC5314 and CBS5736, were evaluated. These strains originated as clinical isolates and SC5314 has been widely used in biochemical, genetic
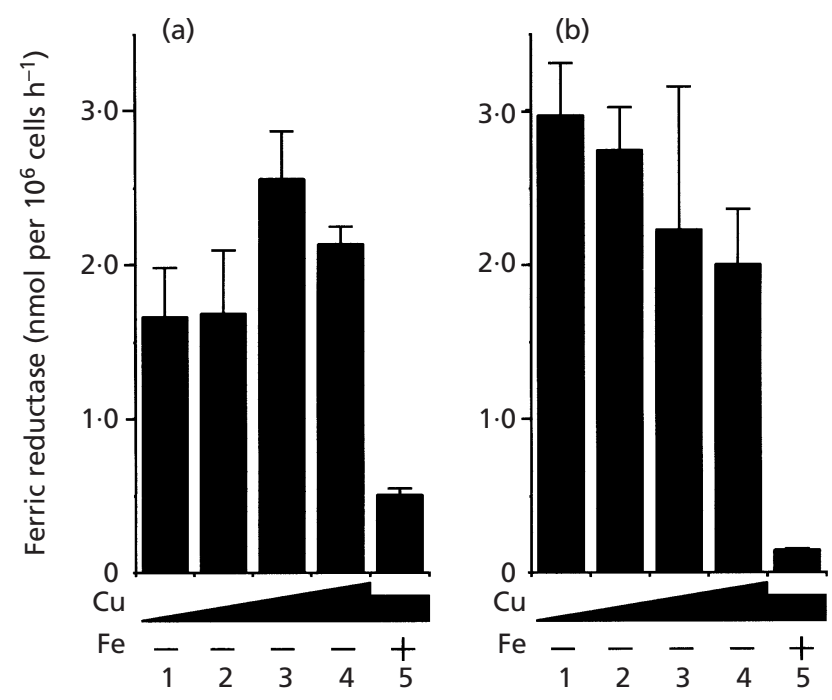

Fig. 1. Regulation of $C$ albicans cell-surface ferric reductase activity. Wild-type C. albicans strains SC5314 (a) and CBS5736 (b) were grown at $30^{\circ} \mathrm{C}$, in defined medium with different concentrations of copper or iron. The copper chelator BCS was added at $1 \mathrm{mM}$ (bar 1) or $0.1 \mathrm{mM}$ (bar 2). No addition (bar 3) or $10 \mu \mathrm{M}$ copper sulfate (bar 4) was added; finally $10 \mu \mathrm{M}$ ferric ammonium sulfate was added (bar 5 ). Cells were grown to exponential-growth phase, washed with $50 \mathrm{mM}$ citrate buffer, $\mathrm{pH} 6.6$, with $5 \%$ D-glucose and resuspended in the same. Cellsurface ferric reductase activity of the cell suspension was assayed. Results are presented as mean values \pm SD for triplicate determinations.

and genomic analyses (Fonzi \& Irwin, 1993). After growth in iron-depleted medium, both strains showed equivalent levels of induced surface ferric reductase activity (Fig. 1a, b). Copper deprivation or copper loading during growth had no effect on ferric reductase activity. However, the addition of $10 \mu \mathrm{M}$ ferric ammonium sulfate decreased ferric reductase activity to $10-20 \%$ of the induced levels.

\section{Copper-dependent high-affinity ferrous iron uptake and copper-independent ferrichrome uptake}

Cells from the same cultures analysed for ferric reductase activity were examined for high-affinity ferrous uptake. Under iron-limiting and copper-replete conditions, ferrous iron uptake activity was 10 -fold higher than that observed for S. cerevisiae (see Fig. 2a, b, bar 3 and data not shown). The effect of copper depletion on high-affinity ferrous iron uptake was examined by growing cells in defined medium containing the copper chelator BCS. The cells were washed free of chelator and ferrous iron uptake was measured (Fig. 2a, b, bars 1 and 2). High-affinity ferrous uptake was abrogated by cellular copper depletion, indicating the presence of a copper-dependent step in iron uptake. The addition of $10 \mu \mathrm{M}$ iron to copper-replete growth medium decreased high-affinity iron uptake to $10-15 \%$ of the induced level (Fig. 2a, b, bar 5). By contrast, iron uptake in SC5314 from the siderophore ferrichrome was not affected by 

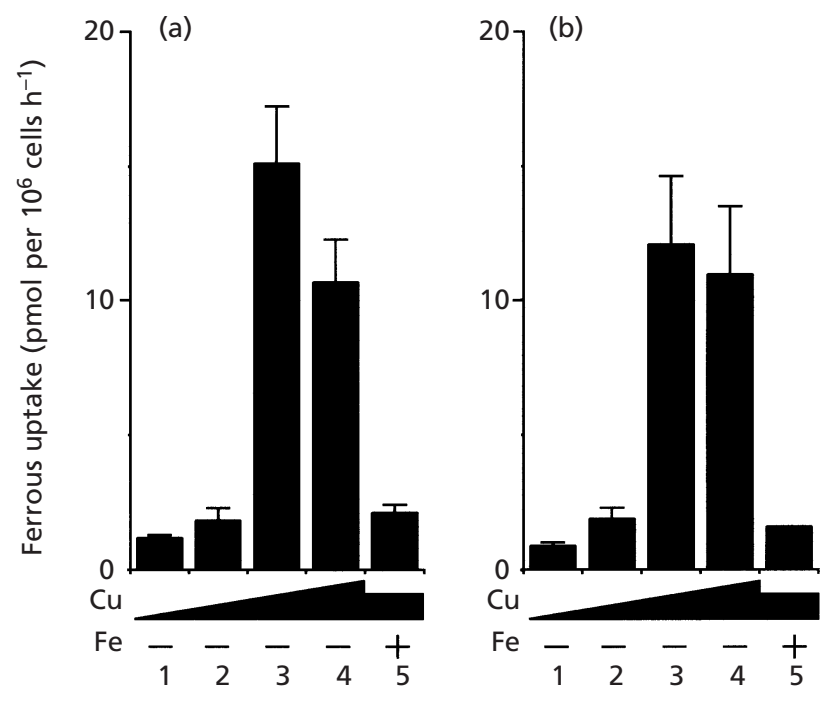

Fig. 2. Regulation of $C$. albicans ferrous iron uptake. Wild-type C. albicans strains SC5314 (a) and CBS5736 (b) were grown as described in the legend to Fig. 1 and cellular ${ }^{55} \mathrm{Fe}$ ferrous uptake was measured. Results are presented as mean values \pm SD for triplicate determinations.

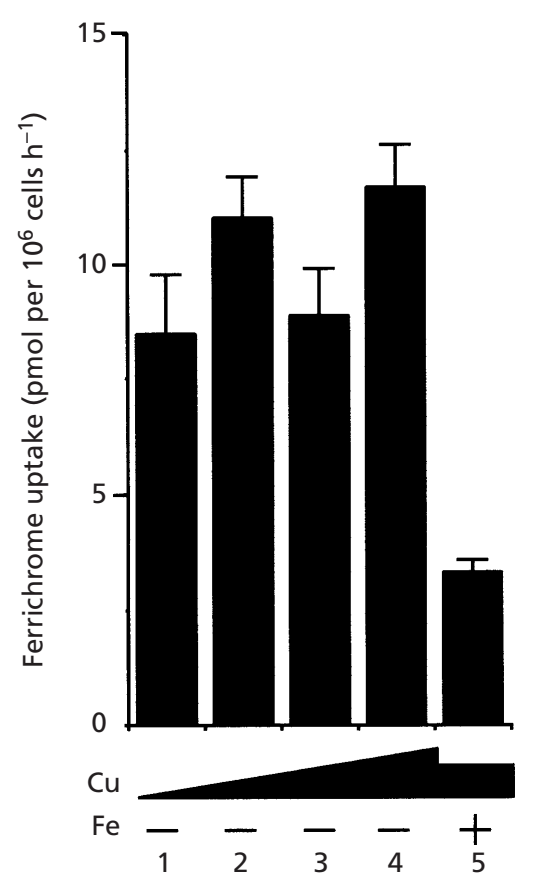

Fig. 3. Regulation of $C$. albicans ferrichrome iron uptake. Wildtype C. albicans strain SC5314 was grown as described in the legend to Fig. 1. The cell suspensions were assayed for cellular ${ }^{55} \mathrm{Fe}$ iron uptake from ferrichrome. Results are presented as mean values \pm SD for triplicate determinations.

copper deprivation (Fig. 3, bars 1 and 2). The addition of iron to the growth medium decreased iron acquisition from ferrichrome to $30-35 \%$ of the induced value (Fig. 3 , bar 5). In summary, both ferrous iron and siderophore iron transport were homeostatically regulated by iron

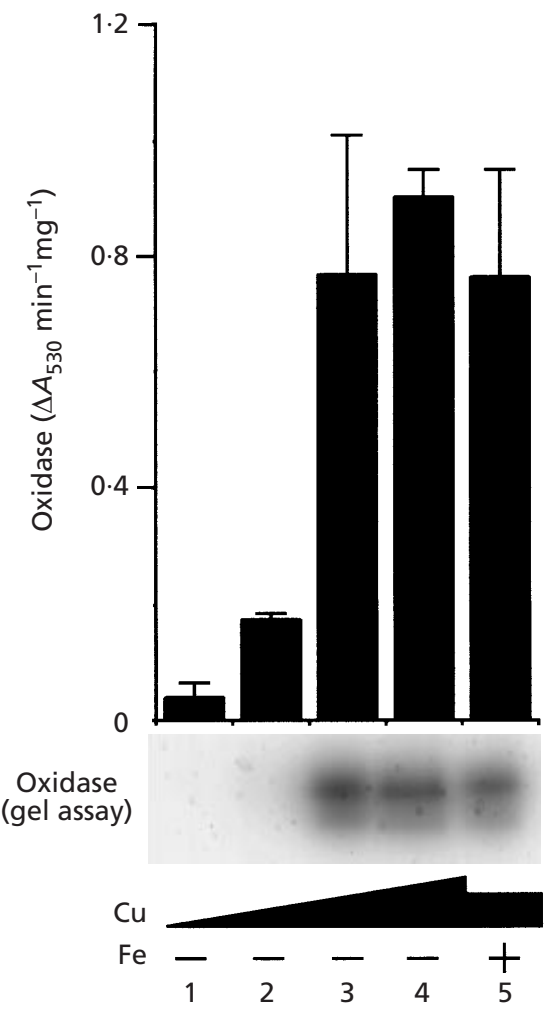

Fig. 4. C. albicans possesses copper-dependent oxidase activity. Wild-type C. albicans strain SC5314 was grown as described in the legend to Fig. 1. Membrane fractions were prepared and assayed for PPD oxidase activity. The mean values of two independent spectrophotometric assays are presented in the column graph and the error bars indicate the higher value. The activity gel assay for PPD oxidase activity of one set of samples is shown below the graph.

exposure during growth. Pathways for iron acquisition were distinguished by the copper requirement for ferrous, but not for siderophore iron transport.

\section{Copper-dependent PPD oxidase activity}

S. cerevisiae contains a plasma membrane multicopper oxidase, encoded by FET3, that is essential for highaffinity ferrous iron transport into cells (Askwith et al., 1994). Activity of this oxidase, which can be assayed using PPD as an artificial substrate, is dependent on copper and delivery of copper into the secretory pathway (Yuan et al., 1995). As noted above, we observed copper dependence of ferrous transport for C. albicans. We also investigated whether the PPD oxidase was copperdependent. Cells were grown under varying metal conditions and membrane fractions were isolated and solubilized in Triton X-100. PPD oxidase activity was evaluated by gel electrophoresis and activity staining or by liquid phase assay. Oxidase activity was observed only in the cultures containing copper. Copper starvation produced by addition of copper chelator virtually abolished oxidase activity (Fig. 4). The parallel requirement of copper for oxidase activity and ferrous uptake suggests that a multicopper oxidase may be 
involved in a reductive pathway of iron uptake in $C$. albicans, analogous to $S$. cerevisiae. Addition of iron had little effect on total membrane oxidase activity in contrast to the repressing effect on cellular iron uptake. However, the C. albicans genome contains five homologues of the $S$. cerevisiae FET3 gene (see below). Therefore, the lack of iron regulation of oxidase activity in the biochemical assays may reflect lack of iron regulation of one or more of these homologous genes.

\section{Cloning of CFL95 by complementation of a ferric reductase mutant of $S$. cerevisiae}

To identify genes involved in iron uptake in C. albicans, we sought to complement mutants of $S$. cerevisiae with a C. albicans genomic library, a strategy that has been utilized by others (Gillum et al., 1984; Kohler \& Fink, 1996; Prasad et al., 1995). C. albicans is an obligate diploid, making identification of mutant phenotypes more difficult, whereas $S$. cerevisiae, a closely related yeast, can be maintained as a haploid. Many genes of $C$. albicans function in $S$. cerevisiae and mutants of the latter with clearly defined phenotypes exist for ferric reductase, multicopper oxidase and ferrous permease. A strain of S. cerevisiae lacking cell-surface ferric reductase activity was transformed with a genomic library from $C$. albicans. Five transformants were identified with enhanced growth on iron-chelated plates and recovery of reductase activity. Although the $C$. albicans genome contains multiple homologues with resemblance to ferric reductases, sequence analysis of the complementing clones revealed that they shared a single ORF of $2 \cdot 1 \mathrm{~kb}$. The corresponding gene, referred to as CFL95 (Candida ferric reductase-like) by the C. albicans genomic sequencing project, exhibited some homology to the $S$. cerevisiae FRE1 and FRE2 ferric reductase genes. The level of amino acid identity was 25 and $28 \%$ with FRE1 and FRE2 proteins, respectively, and was confined to motifs predicted to mediate cofactor interactions. Histidines implicated in haem coordination at residues 309, 324, 379 and 393 were present, as was the HPFT sequence at 471-473 for FAD-isoalloxazine binding and conserved hydrophobic stretches (Shatwell et al., 1996). The corresponding transcript, previously identified as a target of TUP1 repression has been referred to as RBT2 (repressed by TUP1) (Braun et al., 2000). The CFL95 ORF was placed behind by the strong phosphoglyerol kinase (PGK) promoter and transformed into the $\Delta$ fre $1 \Delta$ fre 2 mutant of $S$. cerevisiae lacking reductase activity. The transformants expressed high levels of surface ferric reductase activity approximately 10 -fold more than the wild-type $S$. cerevisiae strain (Fig. 5a), confirming that the CFL95 ORF conferred ferric reductase activity.

\section{Cloning of CaFTR2 by complementation of a ferrous transport mutant of $S$. cerevisiae}

S. cerevisiae lacking the iron permease gene FTR1 grows extremely slowly on agar plates supplemented with the iron chelator ferrozine (Stearman et al., 1996). This mutant strain was transformed with the C. albicans
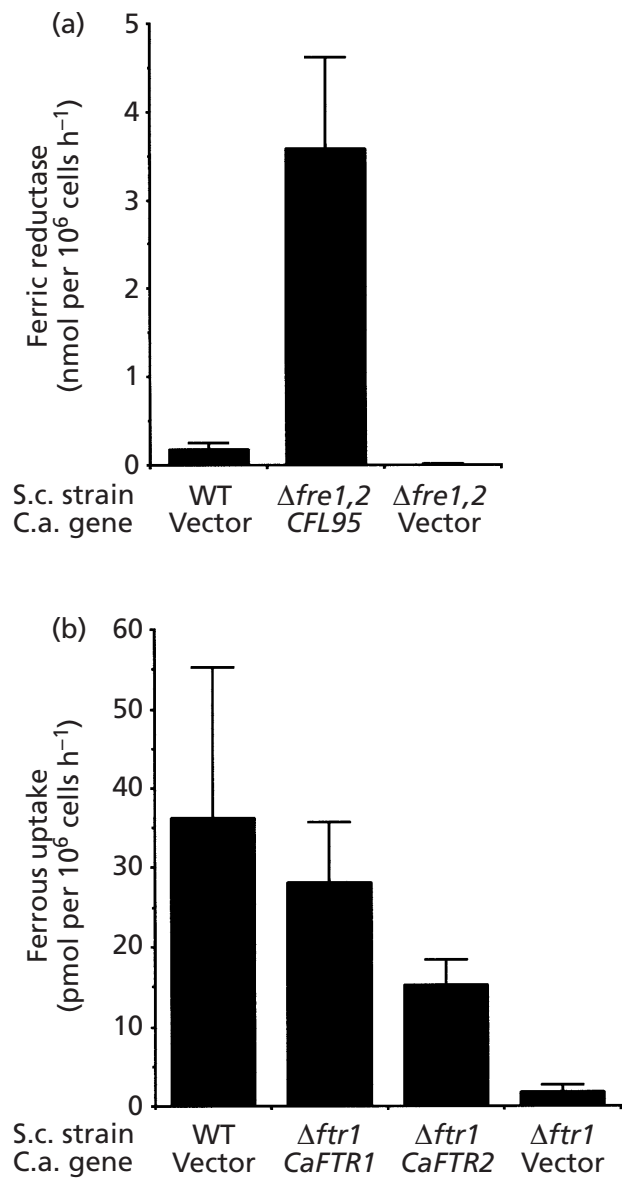

Fig. 5. C. albicans iron acquisition genes function in $S$. cerevisiae. (a) CFL95 ORF under the control of the S. cerevisiae PGK promoter was transformed into the reductase-deficient mutant of $S$. cerevisiae YPH499 $\Delta 1 \Delta 2(\Delta$ fre 1,2$)$. For controls the empty vector was transformed into YPH499 (WT) or mutant $(\Delta$ fre 1,2). Transformants were assayed for ferric reductase activity. (b) CaFTR1 or CaFTR2 ORFs, each under the control of the $S$. cerevisiae PGK promoter, were transformed into a mutant of $S$. cerevisiae $42-3 C 1$ lacking the iron permease $(\Delta f t r 1)$. For controls the empty vector was transformed into YPH499 (WT) or mutant ( $\Delta$ ftri). Transformants were assayed for ferrous iron transport activity.

library and two transformants were selected that grew in the presence of chelator. The plasmids were rescued and found to contain a common $2 \cdot 2 \mathrm{~kb}$ genomic fragment. Sequencing revealed a $1.1 \mathrm{~kb}$ ORF identical to CaFTR2 (Ramanan \& Wang, 2000) with the exception of a single silent nucleotide substitution of $\mathrm{C}$ for $\mathrm{T}$ at position 552 of the coding region. While analysing the flanking regions of CaFTR2, we noted the presence of a highly homologous ORF in the genome database located $18 \cdot 2 \mathrm{~kb}$ away on the opposite DNA strand. The ORF for this gene, referred to as CaFTR 1 in a recent publication (Ramanan \& Wang, 2000), was $83 \%$ identical with CaFTR2 at the nucleotide level and $87 \%$ identical at the amino acid level. The sequence similarity was restricted to the coding regions and did not extend to the immediate $5^{\prime}$ and $3^{\prime}$ untranslated regions or flanking regions. In these flanking regions, no significant se- 


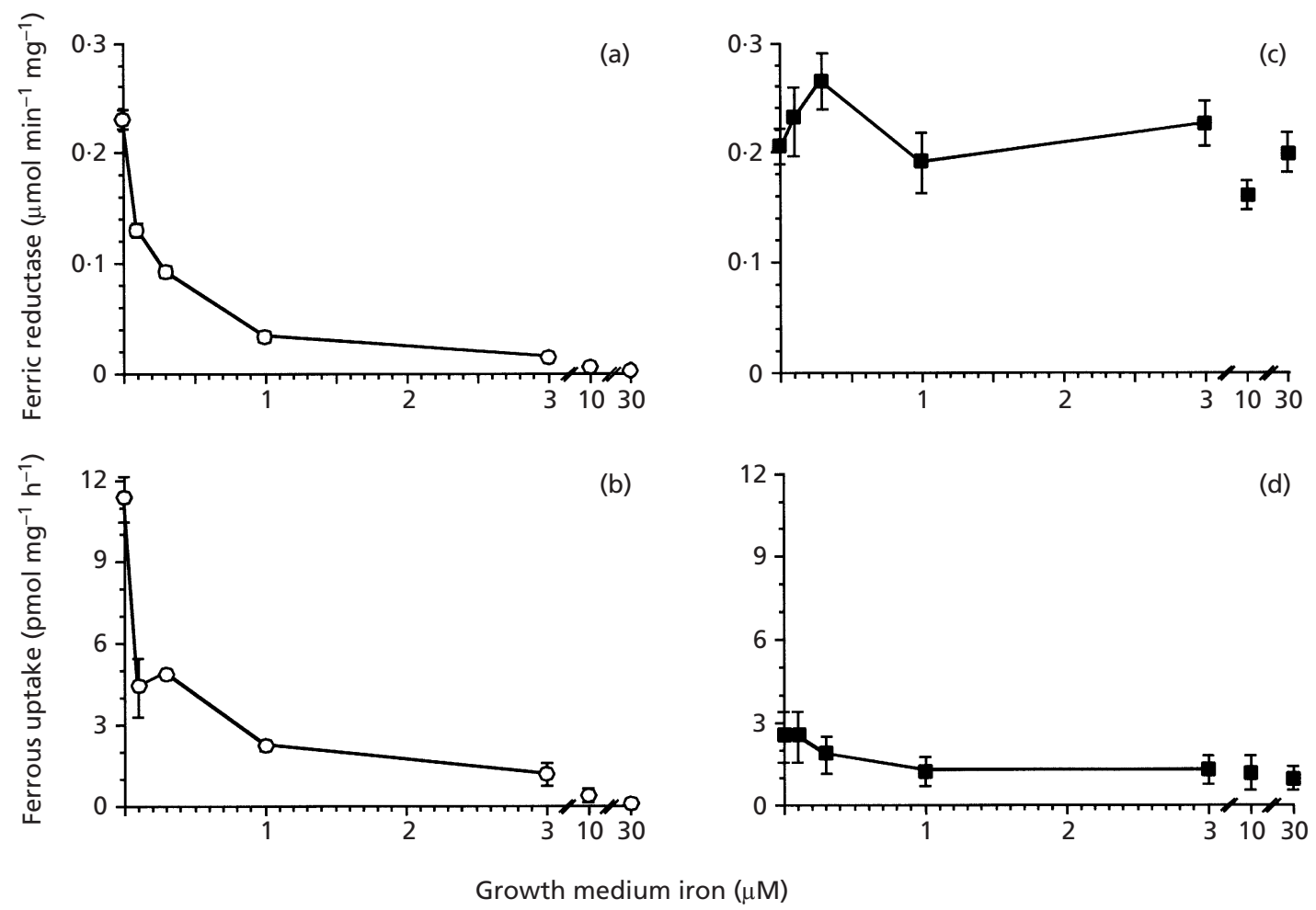

Fig. 6. TUP1 is required for iron-dependent regulation of cell-surface ferric reductase activity and ferrous iron uptake in C. albicans. Wild-type C. albicans strain SC5314 (a and b) and the congenic $\Delta$ tup $1 / \Delta$ tup1 mutant (BCa02-10) (c and d) were grown overnight in low-iron defined medium containing $1 \mu \mathrm{M}$ copper sulfate and iron (ferric ammonium sulfate) as indicated. The cells were diluted into fresh medium and grown for $4 \mathrm{~h}$. Cells were washed and assayed for cell-surface ferric reductase activity ( $a$ and $c$ ) or high-affinity ferrous iron uptake ( $b$ and $d$ ). Each point shows the mean value \pm SD of six assays.

quence similarities were detected by pairwise BLAST at default settings (www.ncbi.nlm.nih.gov/blast/). We amplified the CaFTR1 ORF from genomic DNA by PCR and used it for further studies.

The $\Delta f t r 1$ strain of $S$. cerevisiae exhibited negligible high-affinity iron uptake (Fig. 5b). Expression of CaFTR1 from the PGK promoter in this strain restored high-affinity iron uptake to $77 \%$ of the $S$. cerevisiae wild-type level. Expression of CaFTR2 in a parallel construct increased high-affinity iron uptake to $42 \%$ of the wild-type level (Fig. 5b). Both CaFTR1 and CaFTR2 were able to mediate ferrous uptake into cells, although CaFTR1 exhibited more efficient complementing activity than CaFTR2 (Fig. 5b). The function of Ftr1p in $S$. cerevisiae requires assembly with the multicopper oxidase partner protein Fet3p, and thus CaFTR1 and CaFTR 2 very likely encode proteins capable of assembly and functional interaction with the heterologous Fet3p. Complementation was incomplete in contrast to the reductase complementation, perhaps because of the added constraint for Fet3p interaction.

\section{CaFET99, a candidate multicopper oxidase gene}

A mutant of $S$. cerevisiae deleted for FET3, which like the FTR1 mutant grows slowly on iron-chelated medium, was transformed with the C. albicans library.
However, no complementing clones were identified after screening 50000 transformants or the equivalent of multiple genome coverage. The genome sequence database for C. albicans contains five genes with significant homology to FET3, the multicopper oxidase of $S$. cerevisiae implicated in iron uptake. CaFet $99 \mathrm{p}$ bears the highest homology to $S$. cerevisiae Fet 3 p $(60 \%$ identity, $74 \%$ similarity), retaining all of the copper-binding sites, a putative iron-binding site (Bonaccorsi di Patti et al., 1999), as well as 10 of 14 potential $\mathrm{N}$-glycoslylation sites. Therefore, we amplified this gene directly from genomic DNA and cloned the ORF for further studies. The CaFET99 ORF was unable to complement the FET3 deletion strain of $S$. cerevisiae. We then noticed that two CUG codons were present in the CaFET99 ORF. These would be decoded as leucine in S. cerevisiae and as serine in C. albicans, because of non-classical codon usage in the latter organism. Therefore, the codons in question were changed by site-directed mutagenesis (CUG changed to AGC and TCT, respectively) and the modified clone was retested for complementing activity. Complementing activity again was not conferred. Co-expression with CaFTR1 or CaFTR2 likewise did not confer complementation. The reason for the inability of CaFET99 to complement the $\Delta$ fet 3 mutant of $S$. cerevisiae remains unexplained. Nonetheless, C. albicans possesses PPD oxidase activity and five 


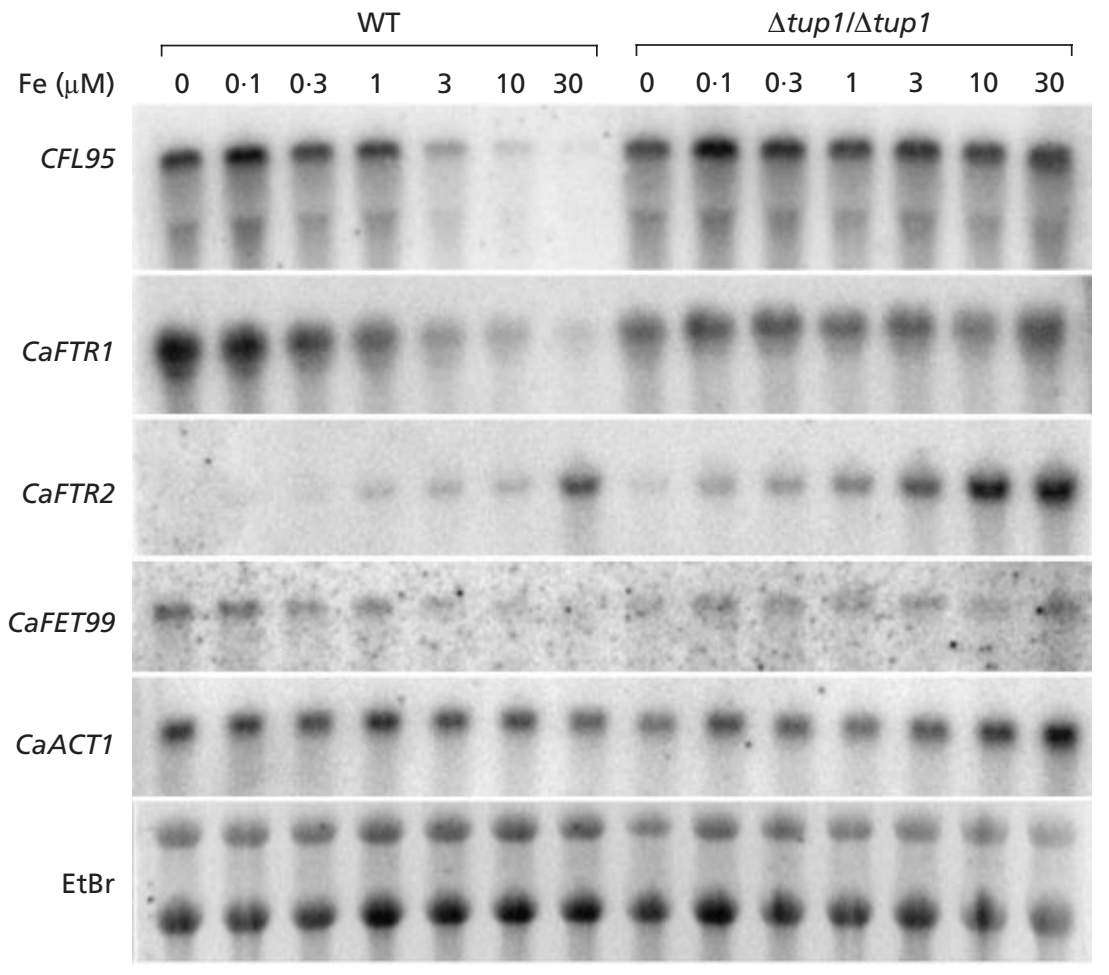

Fig. 7. Expression of genes involved in iron acquisition is misregulated in the $\Delta$ tup1/ $\Delta$ tup1 mutant. Wild-type C. albicans strain SC5314 and the congenic $\Delta$ tup $1 / \Delta$ tup 1 mutant (BCa02-10) were grown in defined medium with varying amounts of iron (as described in the legend to Fig. 6). Total RNA ( $30 \mu \mathrm{g}$ per lane) was isolated and analysed by Northern blotting with probes for the indicated genes. homologous genes belonging to the multicopper oxidase family.

\section{TUP1 required for iron regulation of ferric reductase and ferrous transport activities}

TUP1 of C. albicans functions as a repressor of gene expression. TUP1 mutants grow as filamentous pseudohyphae, indicating a role in repressing genes involved in morphologic change. A role in iron metabolism was suggested by the identification of CFL95, the ferric reductase gene (also called RBT2), as a target of TUP1mediated repression. We therefore decided to compare wild-type and homozygous $\Delta t u p 1 / \Delta t u p 1$ deletion strains for cell-surface ferric reductase activity and highaffinity ferrous iron uptake.

The $\Delta t u p 1 / \Delta t u p 1$ mutant of C. albicans BCa02-10 grew entirely in filamentous form as reported by Braun \& Johnson (2000) and this form was unaltered by manipulations of medium iron availability. Microscopic examination revealed elongated branching forms consisting of discrete septated cells (pseudohyphae), presenting difficulties in expressing ferric reductase and ferrous iron uptake activities in terms of cell number. Instead, for both wild-type and the $\Delta t u p 1 / \Delta t u p 1$ mutant, assays performed on whole cells were expressed on the basis of cellular protein (see Methods). Surprisingly, effects of

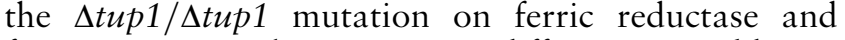
ferrous iron uptake were quite different. In wild-type cells, ferric reductase activity was progressively downregulated by addition of iron to the growth medium (Fig. 6a). Addition of $0 \cdot 1 \mu \mathrm{M}$ iron decreased activity to almost $50 \%$ and $30 \mu \mathrm{M}$ iron decreased activity to $1 \%$ compared to cells grown in the absence of iron (Fig. 6a). In contrast, ferric reductase activity of $\Delta t u p 1 / \Delta t u p 1$ cells was constitutively elevated and unaffected by iron exposure (Fig. 6c). Ferrous iron uptake in the wild-type strain, like ferric reductase, was progressively repressed by iron exposure during growth (Fig. 6b). However, ferrous iron uptake in the $\Delta t u p 1 / \Delta t u p 1$ mutant, while no longer responsive to iron exposures, was neither maximally induced nor repressed (Fig. 6d).

\section{TUP1 required for iron regulation of key transcript levels}

The pathway by which iron availability in the medium is transduced into changes in cellular iron uptake might involve regulated changes in mRNA, proteins or activities of critical elements of the iron uptake system. As a first step to studying iron regulation, transcript levels for candidate genes involved in iron uptake were examined. Transcripts for the ferric reductase gene CFL95 were examined in wild-type C. albicans by Northern blotting. In these cells CFL95 mRNA levels declined as iron concentrations in the growth medium increased (Fig. 7). For the most part, CFL95 mRNA abundance and ferric reductase activity declined in parallel with increasing iron exposure of the cells. An exception was noticed in cells exposed to very low levels of iron in the medium $(0 \cdot 1,0 \cdot 3$ and $1 \mu \mathrm{M}$, Fig. 6a). Under these conditions, ferric reductase activity was repressed, whereas CFL95 mRNA levels were not substantially altered (Fig. 7). Iron-dependent repression of CFL95 mRNA translation, iron-dependent protein turnover, 
(a)
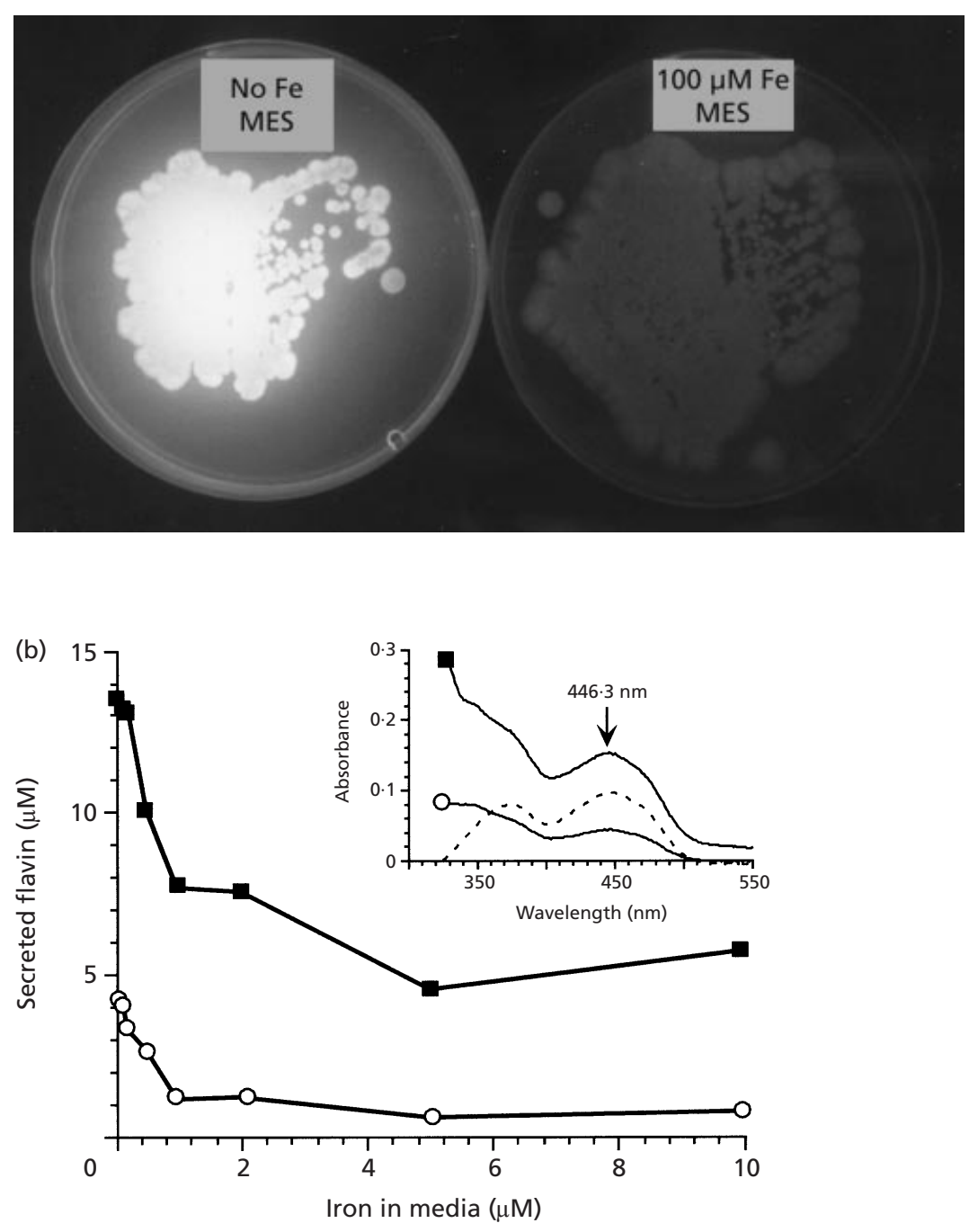

Fig. 8. Flavin secretion by C. albicans: regulation by iron and repression by TUP1. (a) Wild-type C. albicans strain SC5314 was grown on solid agar defined medium containing $50 \mathrm{mM}$ MES, pH 6.2, $1 \mu \mathrm{M}$ copper sulfate and $1 \mathrm{mM}$ ferrozine with no added iron (left) or with $100 \mu \mathrm{M}$ ferric ammonium sulfate (right). After incubation for $3 \mathrm{~d}$ at $30{ }^{\circ} \mathrm{C}$ the plates were photographed under a UV lamp. The iron-chelated culture (left) emitted a bright yellow colour. (b) Wildtype C. albicans strain SC5314 (O) and

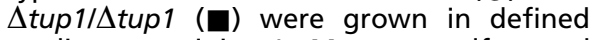
medium containing $1 \mu \mathrm{M}$ copper sulfate and with iron additions as indicated. After $40 \mathrm{~h}$ at $30{ }^{\circ} \mathrm{C}$ the cell cultures were centrifuged to pellet the cells $(1500 \mathrm{~g}, 10 \mathrm{~min})$ and supernatants were assayed for flavin concentration. The insert shows an absorbance scan of conditioned medium from wild-type $(\boldsymbol{\square})$ and $\Delta$ tup $1 / \Delta$ tup $1(O)$ cells grown in low iron medium. A scan for $10 \mu \mathrm{M}$ riboflavin is shown as a dashed line and the $446.3 \mathrm{~nm}$ maximum is indicated. repression of enzymic activity or effects on protein partners could account for these post-transcriptional effects. CFL1, another C. albicans gene able to confer reductase activity to a $\Delta$ fre $1 \Delta$ fre 2 mutant of $S$. cerevisiae (Hammacott et al., 2000), was not detected by Northern blotting under iron-starved or iron-replete conditions (data not shown) and so conclusions could not be drawn regarding its regulation.

CaFTR1 mRNA was also down-regulated by iron exposure during growth (Fig. 7). The decline was progressive with increasing iron addition and correlated well with progressive decreases in ferrous uptake activity (Fig. 6b). By contrast, CaFTR2 levels increased with increasing concentrations of iron in the growth medium. A large increase in mRNA abundance was seen between 10 and $30 \mu \mathrm{M}$ iron. These results are consistent with previous observations regarding the bidirectional regulation of the two C. albicans FTR1 orthologues.

In C. albicans, five multicopper oxidase homologues are present in the genome. CaFET99 was investigated because it showed the highest level of sequence identity to $S$. cerevisiae Fet 3 p $(60 \%$ amino acid identity over the entire protein). Similar to CaFTR1 regulation, the levels of CaFET99 mRNA progressively decreased with addition of iron to the growth medium (Fig. 7). CaFET99 transcripts required a $6 \mathrm{~d}$ exposure for visualization by Northern blotting versus $1 \mathrm{~d}$ for the CaFTR 1 and CaFTR2 transcripts. The decreased signal could represent decreased mRNA abundance for this gene or decreased reactivity of the probe. The probe was directed to a small region $3^{\prime}$ of the ORF, and the precise margins of the $3^{\prime}$ untranslated region have not yet been mapped. Another multicopper oxidase homologue, CaFET3, with $56 \%$ amino acid identity to Fet 3 p of $S$. cerevisiae, was not detected by Northern blotting with a probe directed to the $3^{\prime}$ untranslated region of that gene (data not shown and Eck et al., 1999).

To assess TUP1 effects on iron regulation, total RNA was isolated from the $\Delta t u p 1 / \Delta t u p 1$ strain grown under different iron conditions and probed to analyse ex- 
pression of various genes implicated in iron uptake (Fig. 7). Surprisingly, although in each case iron-dependent regulation was blunted or abolished, the effects were not the same for each gene. CFL95 mRNA levels were maximally induced and unresponsive to iron exposure. These results correlated with the constitutively high ferric reductase activities of the $\Delta t u p 1 / \Delta t u p 1$ strain (Fig. 6c). By contrast, CaFTR1 mRNA in the $\Delta t u p 1 / \Delta t u p 1$ strain was expressed at a medium level, much less than the maximally induced level observed in wild-type cells grown in the absence of iron. Iron-dependent changes were abrogated. These results correlated well with the ferrous uptake data, which also showed mid-level unregulated activities (Fig. 6d). CaFET99 transcripts in the $\Delta t u p 1 / \Delta t u p 1$ strain were present at moderately induced levels (see $1 \mu \mathrm{M}$ level for comparison) and irondependent changes were absent.

The iron response profile of the CaFTR2 transcripts in the $\Delta t u p 1 / \Delta t u p 1$ mutant was unique (Fig. 7). CaFTR2 expression was induced by iron exposure in the wildtype, with a large step increase at $30 \mu \mathrm{M}$ medium iron. In the $\Delta t u p 1 / \Delta t u p 1$ strain, the induction threshold was shifted to a much lower iron level (compare CaFTR2 mRNA level at $30 \mu \mathrm{M}$ in the wild-type with $3 \mu \mathrm{M}$ in the mutant). Furthermore, the large step increase of the wild-type was not seen, but rather a graded increase from 0 to $30 \mu \mathrm{M}$ medium iron. The effects of the $\Delta t u p 1 / \Delta t u p 1$ mutation were to shift downward the threshold for iron-dependent induction of expression of CAFTR 2 and to generally derepress expression. However, iron-dependent expression of the transcripts was preserved over a wide range of medium iron exposures.

CaACT1 transcript levels and ethidium bromide staining of $25 \mathrm{~S}$ and $18 \mathrm{~S}$ rRNAs served as loading controls for these experiments.

\section{Regulation of flavin secretion: role of iron and TUP1}

During the course of these experiments, we observed an intense yellow-green colour of the culture medium from stationary-phase iron-limited cultures of C. albicans. The medium (not shown) and colonies (Fig. 8a) also exhibited bright yellow fluorescence in response to UV excitation. The visible absorbance spectrum of the secreted pigment exhibited a peak at $446.3 \mathrm{~nm}$ (Fig. $8 \mathrm{~b}$ insert), suggesting that a flavin was responsible. The concentrations of flavin in the medium decreased with increasing medium iron concentrations up to $1 \mu \mathrm{M}$ (Fig. $8 b)$. Flavin production by the $\Delta t u p 1 / \Delta t u p 1$ mutant was markedly elevated at all growth medium iron concentrations, although some level of iron response was maintained (Fig. 8b).

\section{DISCUSSION}

C. albicans possesses more than one type of iron acquisition system (Eck et al., 1999; Hammacott et al., 2000; Lesuisse et al., 1998; Manns et al., 1994; Ramanan \& Wang, 2000), perhaps because each system is specifically adapted for an environmental niche or because redundancy is desired for this crucial function. As shown

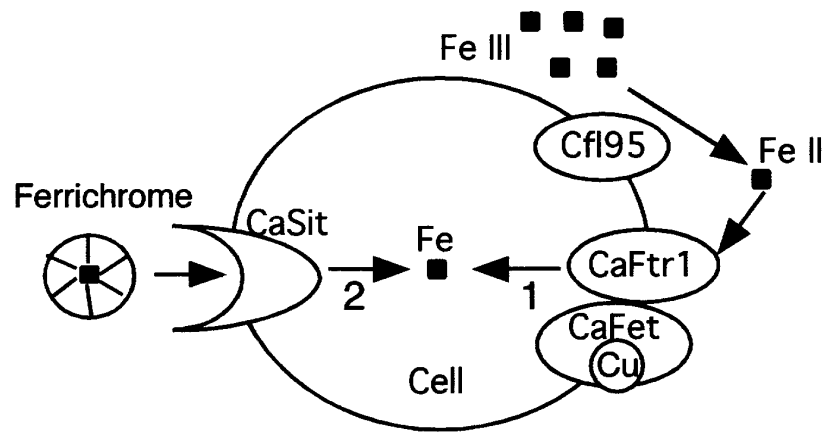

Fig. 9. Model of the iron acquisition systems of C. albicans. 1. The copper-dependent reductive system. A ferric reductase (Cfl95) reduces iron to the ferrous form which is taken into the cell by a complex consisting of the ferrous permease (CaFtr 1 ) and a multicopper oxidase (CaFet). 2. The copper-independent siderophore permease (CaSit) system for ferrichrome uptake.

here, at least two systems can be distinguished: the reductive copper-dependent iron uptake of elemental iron and a copper-independent siderophore transport system (Fig. 9). In the former, iron uptake from ferric chelates involves a surface reductase encoded by CFL95 and a ferrous permease encoded by CaFTR1. The ferrous transport component is copper-dependent, as is the PPD oxidase activity found in an isolated membrane fraction. Therefore, a multicopper oxidase is likely to be involved, analogous to the situation in $S$. cerevisiae (Askwith et al., 1994; Stearman et al., 1996). The gene or genes responsible for the oxidase activity mediating iron uptake have been difficult to ascertain, because five homologous multicopper oxidase genes exist in the Candida genome and the most homologous, CaFET99, was unable to complement the orthologous mutant of $S$. cerevisiae. The copper dependence of elemental iron uptake contrasts with the copper independence of iron uptake from the siderophore ferrichrome. A single siderophore permease homologue is present in the genome of C. albicans that may mediate ferrichrome uptake (Lesuisse et al., 2001; Ardon et al., 2001), C. albicans may even synthesize its own siderophores (Ismail et al., 1985), providing even greater versatility in scavenging iron.

The reductive iron uptake system of C. albicans resembles that of $S$. cerevisiae, but is distinguished by greater regulatory complexity and the existence of multiple genes for each component. Of special interest are the two forms of the permease, CaFTR1 and CaFTR2 (Ramanan \& Wang, 2000). These are highly homologous within their coding regions but dissimilar in their untranslated and flanking regions, consistent with their very different patterns of expression. CaFTR1 is induced by iron deprivation while CaFTR 2 is induced by iron exposure. A conundrum is raised by the complementation data presented here. Both genes are able to complement the orthologous $\Delta f t r 1$ mutant of $S$. cerevisiae by conferring high-affinity iron uptake activity upon this mutant. In C. albicans, however, under 
conditions in which CaFTR2 is induced $(>30 \mu \mathrm{M}$ medium iron concentration), high-affinity iron transport into the cell does not occur. The implication is that another level of control exists. Perhaps in this setting, the CaFtr2p functions elsewhere in the cell or with an alternative oxidase (Fet3p) homologue that confers iron sequestering or iron detoxifying activity. Such an activity might be needed under special iron stress conditions.

Regulation of genes involved in iron uptake responds to iron availability, either by down-regulation (CFL95, CaFTR1, CaFET99) or by up-regulation (CaFTR2). The sensor-regulator that transduces changes in available iron into effects on transcription has not been identified for C. albicans. A homologue of AFT1 (Yamaguchi-Iwai et al., 1996), the sensor-regulator in $S$. cerevisiae, exists (http://www-sequence.stanford.edu/ group/candida) and might provide such a function, although no direct information exists regarding the function of this gene. Post-transcriptional iron regulatory effects might also occur as shown by the marked repression of CFL95 that occurs at low iron levels without changes in transcript levels. Mechanisms for these regulatory effects also remain to be defined.

Iron-dependent gene regulation is linked to changes in the morphological growth form of C. albicans by the role of the TUP1 repressor in both processes. In rich laboratory medium, the organism grows as a budding yeast, but in some settings it changes to a filamentous or hyphal growth form (Brown \& Gow, 1999; Ernst, 2000). The ability to effect this change is correlated with virulence (Lo et al., 1997; Mitchell, 1998), and TUP1 maintains the organism in the yeast growth form through repressive effects on numerous target genes (Braun et al., 2000). Expression of CFL95, the ferric reductase gene, was previously shown to be repressed by TUP1 (Braun et al., 2000). Here we show that TUP1 is required for iron-dependent repression of the CFL95 transcript and more generally for correct iron sensing and iron-dependent gene regulation. Extensive characterization of the S. cerevisiae TUP1 homologue shows that the protein does not interact directly with DNA. Instead it is brought to the promoter regions of specific target genes by DNA-interacting regulatory proteins where it acts to shut off transcription (Smith \& Johnson, 2000). TUP1 of S. cerevisiae has been implicated in diverse processes such as mating type silencing, response to nitrogen starvation (Smith \& Johnson, 2000) and regulation of siderophore iron uptake (Lesuisse et al., 2001). In C. albicans, an iron sensor-regulator protein might interact with a specific DNA binding site in the CFL95 promoter region. Interaction of this sensor protein with TUP1 protein in turn might mediate irondependent repression of gene expression. How specific nutritional signals (such as iron availability) are transduced by a general and global repressor protein remains a mysterious and central problem in understanding TUP1 function. Iron-dependent regulation of other genes was also TUP1-dependent, although different effects were discerned. For CaFTR1 and CaFET99, TUP1 was required for iron regulation, although the

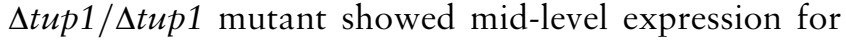
these transcripts, suggesting a requirement of TUP1 for both maximally induced and maximally repressed transcript levels. For CaFTR2, iron-dependent regulation was still observed in the absence of TUP1, although the threshold for induction was shifted downward and the expression level was increased.

During growth of C. albicans in low iron conditions, the medium became yellow-green due to accumulation of flavins. The amount of flavin was inversely related to iron availability in the medium and was markedly increased in the absence of TUP1. Flavins are versatile molecules that can catalyse two-electron dehydrogenations and participate in single-electron reductions (Massey, 2000). The synthesis and excretion of flavins in response to iron deficiency has been observed in other yeasts as well as plants (Fedorovich et al., 1999; Susin et al., 1993). The switch to flavin production might be advantageous by making available an alternative to iron- and cytochrome-dependent electron transport for various metabolic pathways. Alternatively, reduced flavins might facilitate release of iron from ferric chelates or ferrisiderophores, thereby facilitating cellular iron acquisition (Coves \& Fontecave, 1993).

Previous work has shown that inactivation of CaFTR1 leads to decreased killing of mice subjected to intravenous injections of this mutant of C. albicans (Ramanan \& Wang, 2000). Therefore, the copperdependent pathway of iron acquisition involving ferric reductase, multicopper oxidase and ferrous permease must be involved in a critical step in pathogenesis of infections following intravenous dissemination of the organism. Macrophages are mainstays of the circulating immune defence, able to phagocytose Candida in the circulation (Vazquez-Torres \& Balish, 1997). Exposure of C. albicans to the environment of the phagosome apparently causes deprivation for some nutrients, thereby inducing genes of the glyoxylate cycle (Lorenz \& Fink, 2001). Changes in iron availability might occur within the phagosome (Kuhn et al., 1999) and survival of C. albicans might require specific iron-regulated responses. The progression of C. albicans infections can be viewed in terms of complex host-pathogen interactions specific to various niches (De Bernardis et al., 1998). Each niche has peculiar features regarding the form and availability of iron. The regulation and function of iron uptake genes within host niches still needs to be defined and the use of reductive verses siderophore pathways for iron acquisition will require further study. Exploration of these factors may better define the role of iron in virulence and suggest therapeutic approaches.

\section{ACKNOWLEDGEMENTS}

We are grateful to H. Liu and G. Fink for the gift of the C. albicans genomic library. Conversations with Gerry Fink were critical in stimulating our interest in the role of iron in the biology of C. albicans and we are grateful for these conversations. We express our thanks to B. Braun and A. Johnson for the gift of the C. albicans tup1 deletion strains. Some of 
the sequence data for C. albicans were obtained from the Stanford DNA Sequencing and Technology Center website at http://www-sequence.stanford.edu/group/candida, which is supported by funds from the NIDR and the Burroughs Wellcome Fund. This work was supported by the American Cancer Society Grant RPG-00-101-01-MBC and by grants from the French Ministère de la Recherche (Programme de Recherches Fondamentales en Microbiologie, Maladies Infectieuses et Parasitaires and Réseau Infection Fongique).

\section{REFERENCES}

Agatep, R., Kirkpatrick, R. D., Parchaliuk, D. L., Woods, R. A. \& Gietz, R. D. (1998). Transformation of Saccharomyces cerevisiae by the lithium acetate/single-stranded carrier DNA/polyethylene glycol (LiAc/ss-DNA/PEG) protocol. Tech Tips Online (http:// tto.trends.com).

Ardon, O., Bussey, H., Philpott, C., Ward, D. M., Davis-Kaplan, S., Verroneau, S., Jiang, B. \& Kaplan, J. (2001). Identification of a Candida albicans ferrichrome transporter and its characterization by expression in Saccharomyces cerevisiae. J Biol Chem (in press).

Askwith, C. C., Eide, D., Van Ho, A., Bernard, P. S., Li, L., DavisKaplan, S., Sipe, D. M. \& Kaplan, J. (1994). The FET3 gene of $S$. cerevisiae encodes a multicopper oxidase required for ferrous iron uptake. Cell 76, 403-410.

Askwith, C. C., de Silva, D. \& Kaplan, J. (1996). Molecular biology of iron acquisition by Saccharomyces cerevisiae. Mol Microbiol 20, 27-34.

Becker, D. M. \& Guarente, L. (1991). High-efficiency transformation of yeast by electroporation. Methods Enzymol 194, 182-187.

Bonaccorsi di Patti, M. C., Pascarella, S., Catalucci, D. \& Calabrese, L. (1999). Homology modeling of the multicopper oxidase Fet3 gives new insights in the mechanism of iron transport in yeast. Protein Eng 12, 895-897.

Braun, B. R. \& Johnson, A. D. (1997). Control of filament formation in Candida albicans by the transcriptional repressor TUP1. Science 277, 105-109.

Braun, B. R. \& Johnson, A. D. (2000). TUP1, CPH1 and EFG1 make independent contributions to filamentation in Candida albicans. Genetics 155, 57-67.

Braun, B. R., Head, W. S., Wang, M. X. \& Johnson, A. D. (2000). Identification and characterization of TUP1-regulated genes in Candida albicans. Genetics 156, 31-44.

Brown, A. J. \& Gow, N. A. (1999). Regulatory networks controlling Candida albicans morphogenesis. Trends Microbiol 7, 333-338.

Coves, J. \& Fontecave, M. (1993). Reduction and mobilization of iron by a $\mathrm{NAD}(\mathrm{P}) \mathrm{H}$ : flavin oxidoreductase from Escherichia coli. Eur J Biochem 211, 635-641.

Dancis, A., Klausner, R. D., Hinnebusch, A. G. \& Barriocanal, J. G. (1990). Genetic evidence that ferric reductase is required for iron uptake in Saccharomyces cerevisiae. Mol Cell Biol 10, 2294-2301.

Dancis, A., Yuan, D. S., Haile, D., Askwith, C., Eide, D., Moehle, C., Kaplan, J. \& Klausner, R. D. (1994). Molecular characterization of a copper transport protein in S. cerevisiae: an unexpected role for copper in iron transport. Cell 76, 393-402.

De Bernardis, F., Muhlschlegel, F. A., Cassone, A. \& Fonzi, W. A. (1998). The $\mathrm{pH}$ of the host niche controls gene expression in and virulence of Candida albicans. Infect Immun 66, 3317-3325.

Eck, R., Hundt, S., Hartl, A., Roemer, E. \& Kunkel, W. (1999). A multicopper oxidase gene from Candida albicans: cloning, characterization and disruption. Microbiology 145, 2415-2422.
Ernst, J. F. (2000). Transcription factors in Candida albicans environmental control of morphogenesis. Microbiology 146, 1763-1774.

Fedorovich, D., Protchenko, O. \& Lesuisse, E. (1999). Iron uptake by the yeast Pichia guilliermondii. Flavinogenesis and reductive iron assimilation are co-regulated processes. Biometals 12, 295-300.

Finegold, A. A., Shatwell, K. P., Segal, A. W., Klausner, R. D. \& Dancis, A. (1996). Intramembrane bis-heme motif for transmembrane electron transport conserved in a yeast iron reductase and the human NADPH oxidase. J Biol Chem 271, 31021-31024.

Fonzi, W. A. \& Irwin, M. Y. (1993). Isogenic strain construction and gene mapping in Candida albicans. Genetics 134, 717-728.

Fridkin, S. K. \& Jarvis, W. R. (1996). Epidemiology of nosocomial fungal infections. Clin Microbiol Rev 9, 499-511.

Gillum, A. M., Tsay, E. Y. \& Kirsch, D. R. (1984). Isolation of the Candida albicans gene for orotidine- 5 -phosphate decarboxylase by complementation of $S$. cerevisiae ura3 and E. coli pyrF mutations. Mol Gen Genet 198, 179-182.

Hammacott, J. E., Williams, P. H. \& Cashmore, A. M. (2000). Candida albicans CFL1 encodes a functional ferric reductase activity that can rescue a Saccharomyces cerevisiae fre 1 mutant. Microbiology 146, 869-876.

Ismail, A., Bedell, G. W. \& Lupan, D. M. (1985). Siderophore production by the pathogenic yeast, Candida albicans. Biochem Biophys Res Commun 130, 885-891.

Kohler, J. R. \& Fink, G. R. (1996). Candida albicans strains heterozygous and homozygous for mutations in mitogen-activated protein kinase signaling components have defects in hyphal development. Proc Natl Acad Sci U S A 93, 13223-13228.

Kuhn, D. E., Baker, B. D., Lafuse, W. P. \& Zwilling, B. S. (1999). Differential iron transport into phagosomes isolated from the RAW264.7 macrophage cell lines transfected with Nramp1Gly169 or Nramp1Asp169. J Leukoc Biol 66, 113-119.

Lesuisse, E., Simon-Casteras, M. \& Labbe, P. (1998). Siderophoremediated iron uptake in Saccharomyces cerevisiae: the SIT1 gene encodes a ferrioxamine $\mathrm{B}$ permease that belongs to the major facilitator superfamily. Microbiology 144, 3455-3462.

Lesuisse, E., Blaiseau, P. L., Dancis, A. \& Camadro, J.-M. (2001). Siderophore uptake and use by the yeast Saccharomyces cerevisiae. Microbiology 147, 289-298.

Lesuisse, E., Knight, S. A. B., Camadro, J.-M. \& Dancis, A. (2002). Siderophore uptake by Candida albicans: relationship to the dimorphic transition and comparison with Saccharomyces cerevisiae. Yeast (in press).

Liu, H., Kohler, J. \& Fink, G. R. (1994). Suppression of hyphal formation in Candida albicans by mutation of a STE12 homolog. Science 266, 1723-1726; erratum 267, 17.

Lo, H. J., Kohler, J. R., DiDomenico, B., Loebenberg, D., Cacciapuoti, A. \& Fink, G. R. (1997). Nonfilamentous C. albicans mutants are avirulent. Cell 90, 939-949.

Lorenz, M. C. \& Fink, G. R. (2001). The glyoxylate cycle is required for fungal virulence. Nature 412, 83-86.

Manns, J. M., Mosser, D. M. \& Buckley, H. R. (1994). Production of a hemolytic factor by Candida albicans. Infect Immun 62, 5154-5156.

Massey, V. (2000). The chemical and biological versatility of riboflavin. Biochem Soc Trans 28, 283-296.

Mitchell, A. P. (1998). Dimorphism and virulence in Candida albicans. Curr Opin Microbiol 1, 687-692. 
Prasad, R., De Wergifosse, P., Goffeau, A. \& Balzi, E. (1995). Molecular cloning and characterization of a novel gene of Candida albicans, CDR1, conferring multiple resistance to drugs and antifungals. Current Genetics 27, 320-329.

Ramanan, N. \& Wang, Y. (2000). A high-affinity iron permease essential for Candida albicans virulence. Science 288, 1062-1064.

Ratledge, C. \& Dover, L. G. (2000). Iron metabolism in pathogenic bacteria. Annu Rev Microbiol 54, 881-941.

Shatwell, K. P., Dancis, A., Cross, A. R., Klausner, R. D. \& Segal, A. W. (1996). The FRE1 ferric reductase of Saccharomyces cerevisiae is a cytochrome b similar to that of NADPH oxidase. J Biol Chem 271, 14240-14244.

Smith, R. L. \& Johnson, A. D. (2000). Turning genes off by Ssn6Tup1: a conserved system of transcriptional repression in eukaryotes. Trends Biochem Sci 25, 325-330.

Spizzo, T., Byersdorfer, C., Duesterhoeft, S. \& Eide, D. (1997). The yeast FET5 gene encodes a FET3-related multicopper oxidase implicated in iron transport. Mol Gen Genet 256, 547-556.

Stearman, R., Dancis, A. \& Klausner, R. D. (1998). YIpDCE1 - An integrating plasmid for dual constitutive expression in yeast. Gene 212, 197-202.

Stearman, R., Yuan, D. S., Yamaguchi-Iwai, Y., Klausner, R. D. \&
Dancis, A. (1996). A permease-oxidase complex involved in highaffinity iron uptake in yeast. Science 271, 1552-1557.

Susin, S., Abian, J., Sanchez-Baeza, F., Peleato, M. L., Abadia, A., Gelpi, E. \& Abadia, J. (1993). Riboflavin $3^{\prime}$ - and $5^{\prime}$-sulfate, two novel flavins accumulating in the roots of iron-deficient sugar beet (Beta vulgaris). J Biol Chem 268, 20958-20965.

Vazquez-Torres, A. \& Balish, E. (1997). Macrophages in resistance to candidiasis. Microbiol Mol Biol Rev 61, 170-192.

Warnock, D. W. (1998). Fungal infections in neutropenia : current problems and chemotherapeutic control. J Antimicrob Chemother 41 Suppl. D, 95-105.

Yamaguchi-Iwai, Y., Stearman, R., Dancis, A. \& Klausner, R. D. (1996). Iron-regulated DNA binding by the AFT1 protein controls the iron regulon in yeast. EMBO J 15, 3377-3384.

Yuan, D. S., Stearman, R., Dancis, A., Dunn, T., Beeler, T. \& Klausner, R. D. (1995). The Menkes/Wilson disease gene homologue in yeast provides copper to a ceruloplasmin-like oxidase required for iron uptake. Proc Natl Acad Sci U S A 92, 2632-2636.

Received 28 August 2001; revised 1 October 2001; accepted 23 October 2001. 